\title{
Interface Reaction Characterization and Interfacial Effects in Multilayers
}

\author{
Troy W. Barbee, Jr.
}

Mark A. Wall

April 1998

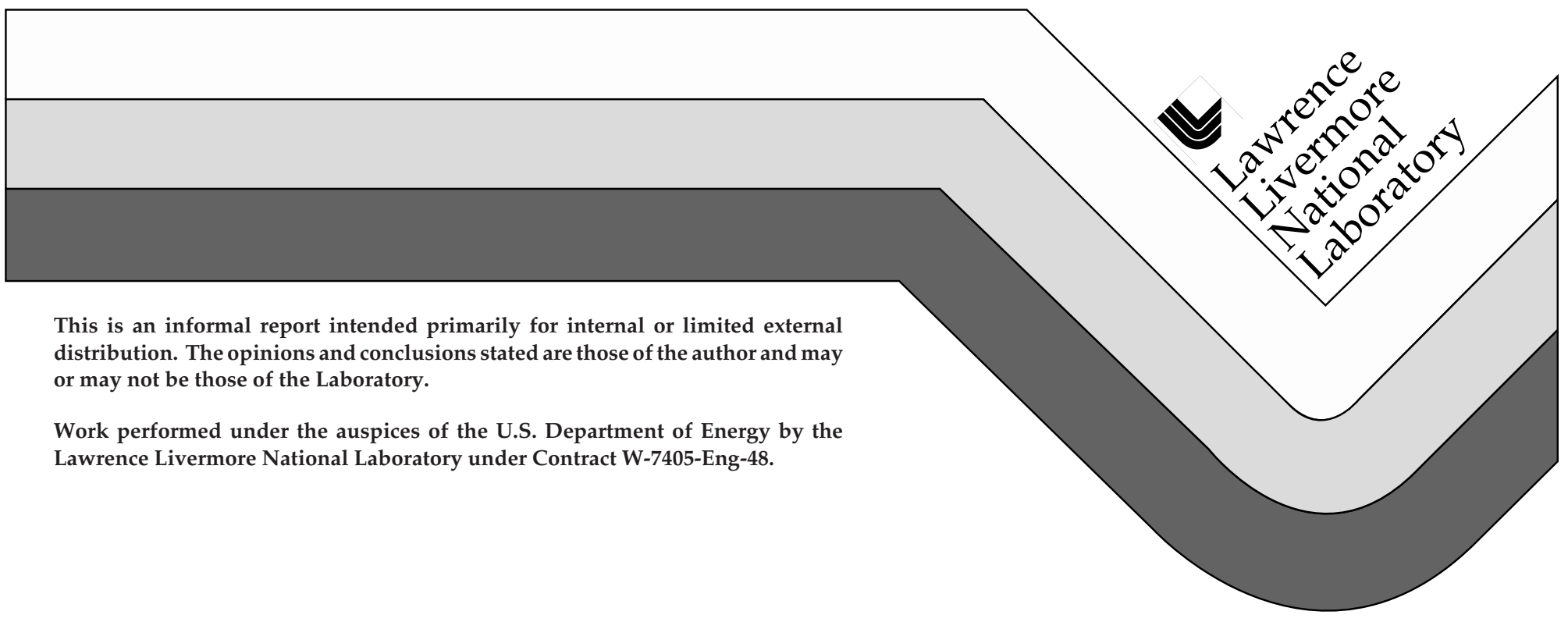




\section{DISCLAIMER}

This document was prepared as an account of work sponsored by an agency of the United States Government. Neither the United States Government nor the University of California nor any of their employees, makes any warranty, express or implied, or assumes any legal liability or responsibility for the accuracy, completeness, or usefulness of any information, apparatus, product, or process disclosed, or represents that its use would not infringe privately owned rights. Reference herein to any specific commercial product, process, or service by trade name, trademark, manufacturer, or otherwise, does not necessarily constitute or imply its endorsement, recommendation, or favoring by the United States Government or the University of California. The views and opinions of authors expressed herein do not necessarily state or reflect those of the United States Government or the University of California, and shall not be used for advertising or product endorsement purposes.

This report has been reproduced directly from the best available copy.

Available to DOE and DOE contractors from the Office of Scientific and Technical Information P.O. Box 62, Oak Ridge, TN 37831

Prices available from (423) 576-8401

Available to the public from the National Technical Information Service

U.S. Department of Commerce 5285 Port Royal Rd., Springfield, VA 22161 


\title{
INTERFACE REACTION CHARACTERIZATION \\ AND

\begin{abstract}
INTERFACIAL EFFECTS IN MULTILAYERS
\end{abstract}

\author{
Troy W. Barbee, Jr. and Mark A. Wall \\ Lawrence Livermore National Laboratory \\ Chemistry and Materials Science Department \\ Livermore, CA 94550
}

\begin{abstract}
The performance of multilayer structures as x-ray, soft x-ray and extreme ultraviolet optics is dependent on the nature of the interfaces between constituent layers. Interfacial structure and the interaction between atoms at interfaces have also been demonstrated to have significant impact on the physical properties of multilayer materials in general and thus on their performance in other applied areas. As short summary of the approaches to characterization of interfaces in multilayer structures is presented as background. Two new techniques for the experimental evaluation of interfacial structure and interfacial structure effects are then considered and examples presented. Model calculations for one of these techniques which support the experimental results are also presented. In conclusion these results are reviewed of and an assessment of their implications relative to multilayer development given.
\end{abstract}

Keywords: multilayers, x-ray optics, interfaces, stability, chemical reactions, transmission electron microscopy,

\section{INTRODUCTION}

Interfacial reactions between the elemental constituents in the component layers of multilayer structures can have a substantial effect on the properties ${ }^{1}$ of these advanced materials. For example, the performance of nano-scale multilayer structures as efficient reflecting X-ray (XR), Soft X-ray (SXR) and Extreme Ultra-Violet (EUV) optic components ${ }^{2,3}$ is strongly dependent on interface factors. Such factors are both intrinsic to the nature of the interfaces between the layers and the materials in the layers as well as extrinsic and determined by the nature of the synthesis process, the environment 
during synthesis and substrate surface defects. The impact of such interfacial factors has been clearly demonstrated in the performance and stability of multilayer $x$-ray optic elements based on the molybdenum-silicon binary alloy system. This material pair is most often used in the Extreme UltraViolet (125 to $340 \AA$ ) where it yields very high reflectivity optics that may be applied in imaging systems to achieve diffraction limited resolution. Characterization and understanding of the interfacial reaction processes and stabilization of interfacial reactions in this material pair as well as in other short wavelength optics material sets appropriate to other spectral domains is thus crucial to the development and application of these nano-structure materials as high efficiency optic elements.

Approaches to experimentally assessing interfacial reactions and interfacial stability are reviewed and experimental results for two new approaches to interface structure effects presented and analyzed. The performance of a multilayer structure fabricated from components selected on the basis one of these new interfacial character characterization techniques are also presented. The paper is concluded with a discussion and summary intended to provide guidance in the development of new multilayer materials.

\section{BACKGROUND}

The earliest general approach to the effects of interface structure on x-ray optic multilayer performance was to measure the reflectivity of the multilayer ${ }^{4}$ and then infer from a total integrated scatter (TIS) analysis a scalar length assumed to be characteristic of interface imperfections. Although this approach allowed comparison of results for different processes or materials little understanding could be gained from the data. The basis for this weakness is that there are several interfacial chemical and structural factors that must be considered whose effects cannot be separated without substantial additional data. The first efforts to provide this additional characterization were several studies of the development of interfacial roughness or atomic interdiffussion as individual films of the components materials to be applied in a multilayer were deposited. The techniques applied in this work were soft SXR scattering ${ }^{5}$ and ellipsometry ${ }^{6,7}$. The results of this work provided substantial guidance to the multilayer optics community and enabled the first stages of the materials engineering of multilayer XR, SXR and EUV optic structures. These efforts provided data that showed that there were materials that exhibited very smooth growth behavior and appeared to have good x-ray optic multilayer performance. Subsequently the primary effort centered on the development of more complete analytical descriptions ${ }^{8}$ of the effects of imperfections. This then enhanced our ability to unfold the magnitude of the effects of imperfections on multilayer performance. More recently high resolution transmission electron microscopy (TEM) $)^{9,10}$ has yielded both quantitative and qualitative information providing substantial guidance to the efforts to develop higher performance multilayer structures. 
There has also been an interesting technique ${ }^{11}$ developed where a layer of one material is deposited and then a layer of longitudinally varying thickness of a second material deposited on the first layer. Auger spectra are then obtained as a function of the thickness of the second layer and provide a measure of the degree and type of chemical reaction at the buried interface.

These physical characterization approaches all test a limited set of elements in a multilayer they study a single interface or a single layer of a multilayer component material and thus only a very small sample of a multilayer. In addition to establishing that a chemical reaction is occurring at the interfaces it is necessary to know the magnitude of the change in the multilayer period resulting from that chemical reaction. On a broader basis, the interference phenomena on which the performance of $x$ ray optic multilayers is based integrates over the full area/volume of the multilayer illuminated. To gain an increased understanding of the impact of imperfections on multilayer performance I believe it is necessary to develop an experimental approach that provides detailed information about interfaces in the multilayer obtained when it is being characterized in a manner directly related to its application. The implementation of two new physical techniques designed to contribute to these two areas is discussed in the next section.

\section{EXPERIMENTAL DESIGN AND SAMPLE FABRICATION}

\subsection{Interfacial Chemical Reaction - Multilayer Period}

The first step is to calibrate the two materials to be used in the multilayer by determining the layer thicknesses deposited for each material as a function of sputter deposition parameters and substrate motion. The multiple period structure is then designed and fabricated so that the sequence in multilayer periods is $\mathrm{d}_{1}: \mathrm{d}_{2}: \mathrm{d}_{3}: \mathrm{d}_{4} ; \mathrm{d}_{5}=1: 2: 4: 8: 16$ starting at the substrate. This structure is then imaged at high magnification in cross-section by TEM. The layer thicknesses expected for each individual layer are known from the initial calibration and the ratios and magnitudes of the resulting multilayer periods from the TEM. This data enables quantitative measurement of the amount of material involved in any interfacial reaction and, as a result of the electron microscopy, an assessment of the structural quality of the interfaces formed. These results enable an evaluation of the potential of a pair of component materials and have the added advantage of establishing a characterization procedure that supports further development of a given pair of multilayer materials.

\subsection{X-ray Standing Wave Fluorescence}

The effects of an unknown interface structure in a multilayer on its properties are often only available through measurements of the multilayers performance. Therefore, access to an experimental technique which enables characterization of interface structural parameters as a direct result of a 
standard x-ray optical measurement such as reflectivity is desirable. It is well known that at the Bragg peak an $x$-ray standing wave is established in a periodic structure such as a multilayer ${ }^{12}$. At fixed $x-$ ray wavelength this standing wave sweeps a distance of half the multilayer period through the multilayer as the Bragg peak is scanned in angle space. The nodes and anti-nodes of this standing wave field sweep over the interfaces and, in principle, provide a probe for sampling the interface structure. Samples were designed to determine the effects of interface imperfection on mutlilayer performance using this characteristic of the diffraction process. The initial objective with these samples was to determine the impact of imperfection position on the two characteristic interfaces on performance. A second objective was to extend the experiments to determine the role of other interface defects if warranted.

Samples with a monolayer thick marker layer deposited at specified interfaces that may be fluoresced by the standing wave field as it sweeps through the mutlilayer are described and experimental results obtained at the Stanford Synchrotron Radiation Laboratory BL - 10 reported. Two multilayer samples, 110 and 111, designed to use the standing wave to sample interface structure were fabricated and characterized. The multilayers were synthesized using standard magnetron sputter deposition techniques from carbon (C), tungsten carbide (WC) and tantalum (Ta) and had 61 periods of $32.28 \AA$ (110) and $32.78 \AA$ (111). The positions of the Ta marker layers are:

Sample 110 - (111) Silicon Substrate/C/Ta/WC/C/Ta/WC/C/Ta/......Ta/WC/C/Ambient

Sample 111 - (111) Silicon Substrate/C/WC/Ta/C/WC/Ta/C/WC/Ta/....../WC/Ta/C/Ambient.

The design layer thicknesses deposited are $t_{\mathrm{C}}=15.9 \AA, \mathrm{t}_{\mathrm{WC}}=15.6 \AA$ and $\mathrm{t}_{\mathrm{Ta}}=2.25 \AA$. It is expected that the Ta reacted with $1.25 \AA$ of the carbon to form $\mathrm{TaC}$ resulting in the observed multilayer periods. The selection of these materials was made on the basis of three materials properties. 1) WC is the highest carbon containing compound in the W/C binary system so that compositionally abrupt and potentially smooth interfaces are expected for this system. 2) It was expected that the Ta would react with the C to form $\mathrm{TaC}$ which is isostructural with WC. 3) Most importantly, the $\mathrm{L}_{\mathrm{III}}$ absorption edges of Ta and $\mathrm{W}$ are $9881 \mathrm{eV}$ and 10,207 eV, respectively. It is therefore possible to measure the reflectivities of these samples at photon energies below, at and above the $\mathrm{L}_{\mathrm{III}}$ edge of $\mathrm{Ta}$ at $9881 \mathrm{eV}$. In these experiments reflectivity was measured at energies of 9850,9879 and $9950 \mathrm{eV}$. Total standing wave fluorescence was also measured by placing a detector above and parallel to the multilayer surface and monitoring the fluorescence intensity as a function of angle of incidence during the reflectivity measurements. The Ta $\mathrm{L}$ absorption only occurs at energies at or above approximately $9881 \mathrm{eV}$ so that scans as described above 
enabled determination of the effects absorbers/imperfections at specific interfaces in this x-ray optic multilayer.

\section{EXPERIMENTAL RESULTS AND MODELING}

\subsection{Interfacial Chemical Reaction - Multilayer Period}

The sample reported on here was designed to have periods of $7 \AA, 14 \AA, 28 \AA, 56 \AA$ and $112 \AA$ and consisted of 248, 124, 62, 31, and 16 layer pairs, respectively, of the above dimensions. The materials selected were molybdenum carbide $\left(\mathrm{Mo}_{2} \mathrm{C}\right)$ and amorphous silicon ( $\mathrm{Si}$ ). Model calculations indicated that the loss in reflectivity resulting from the carbon was minimal - about $2 \%$. Also, it was expected that the $\mathrm{Mo}_{2} \mathrm{C}$ would react with the $\mathrm{Si}$ to form a $\mathrm{Mo}_{x} \mathrm{Si}_{\mathrm{y}} \mathrm{C}_{\mathrm{z}}$ compound blocking the growth of an interfacial reaction layer. Multilayer structures were observable in the TEM for the four longest period segments, as shown in Figure 1. The interface quality is very good with no discernible intermediate chemical reaction layer at the interfaces. Figure 2 shows small sections (10 periods) of the four multilayer structures magnified to produce images that would be identical if no interfacial reaction occurred with line scans of the images. The periods are all smaller than the design values given above by an average of $2.4 \AA$ or $1.2 \AA$ /interface. The nearly vertical lines at the ends of the line scans converge top to bottom in Figure 2. This reflects the fact that $2.4 \AA$ is a much larger fraction of a $14 \AA$ period than a $112 \AA$ period so the total film thickness is smaller i.e. the lines converge. Very high resolution TEM has shown that the $\mathrm{Mo}_{2} \mathrm{C}$ is micro-crystalline and the interfaces are very smooth and abrupt in electron contrast. These results indicated that this materials pair had the potential to yield very high quality multilayers.

$\mathrm{Mo}_{2} \mathrm{C}$-Si multilayers, operating at near normal incidence over the wavelength range $130 \AA$ to $320 \AA$ with $\gamma$ values ranging from 0.13 to 0.5 have been fabricated and characterized. At this time the multilayers are generally observed to have efficiencies above $88 \%$ of that calculated for a perfect multilayer. In several cases performance essentially equal to that predicted for a perfect structure was observed. Figure 3 compares reflectivities measured at two laboratories on two separate samples deposited on 1 in. dia. super polished fused silica substrates in the same sputtering run to calculations for an ideal structure. The agreement is excellent. We are pursuing this observation to gain sufficient understanding to enable generalization to other materials as well as the ability to routinely reproduce this result.

\subsection{X-ray Standing Wave Fluorescence Characterization}

X-ray reflectivity and standing wave experiments on multilayer samples are performed at extreme grazing angles of incidence ( 0 to $3 \mathrm{deg}$.) by scanning angle at fixed photon energy or by scanning 


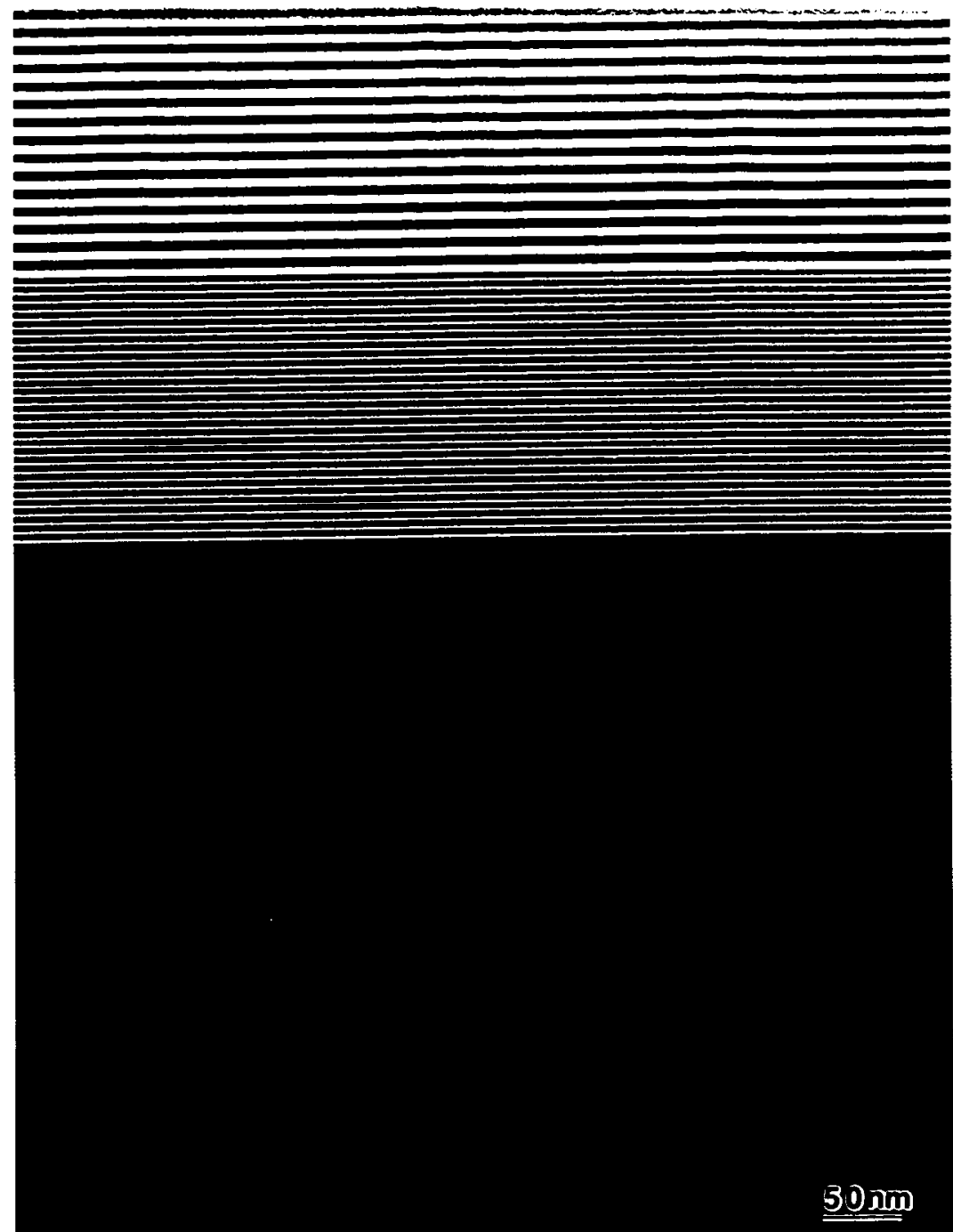

Figure 1. Transmission electron microscopy image of a crosssection of a multi-period multilayer synthesized by magnetron sputter deposition on a 1" dia. super-polished fused silica substrate.
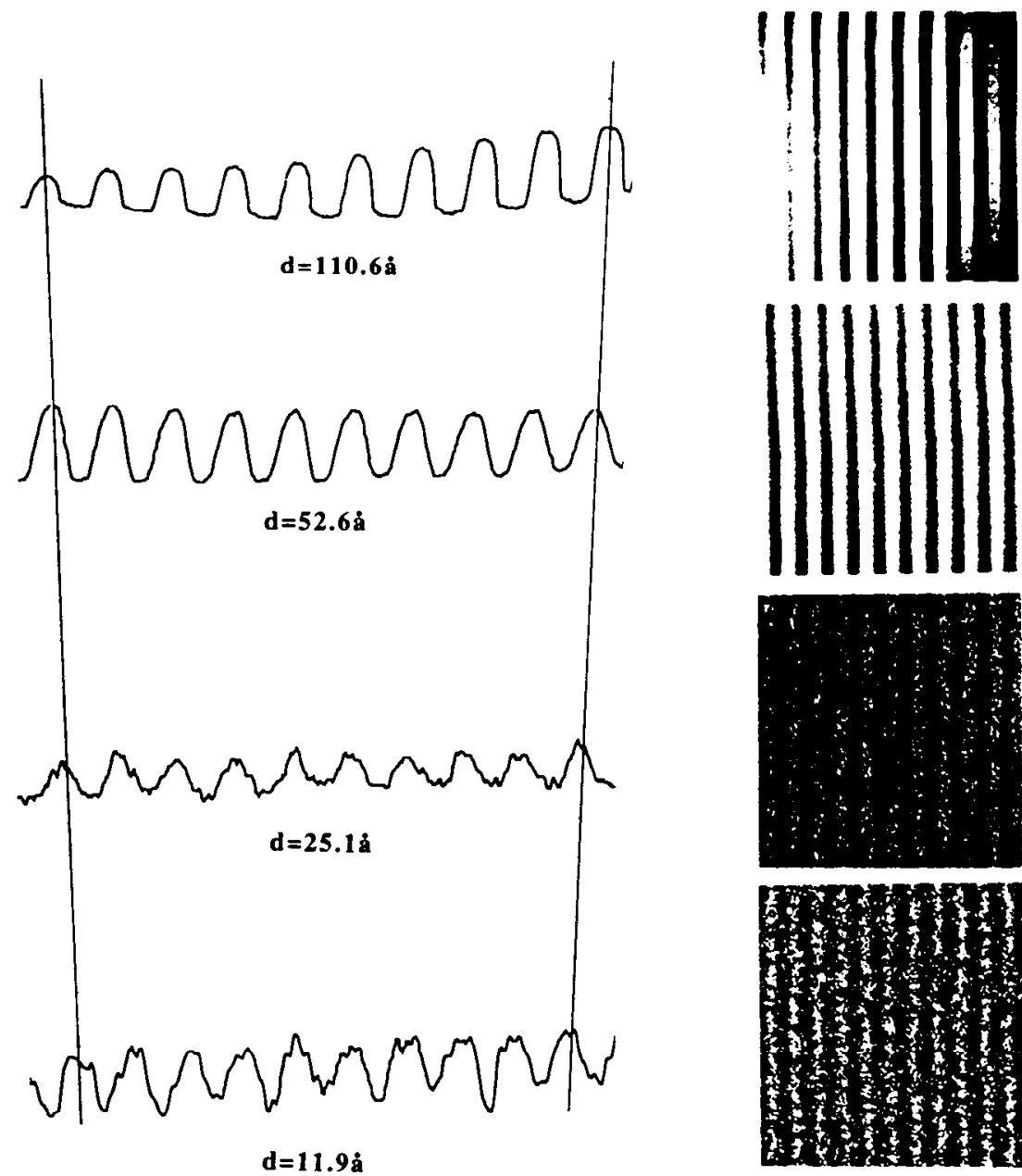

$(1 \mathrm{X})$

$(2 \mathrm{X})$

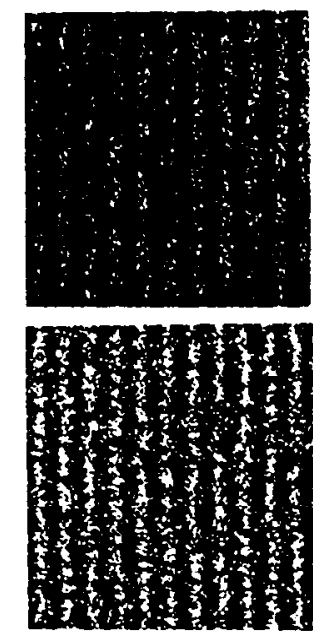

$(4 \mathbf{X})$

Figure 2. Small sections (10 periods) of the four multilayer structures magnified to produce images that would be identical if no interfacial reaction occurred are shown in with line scans of the images. If no interfacial reaction occurred all these 10 period images would be exactly the same size. 


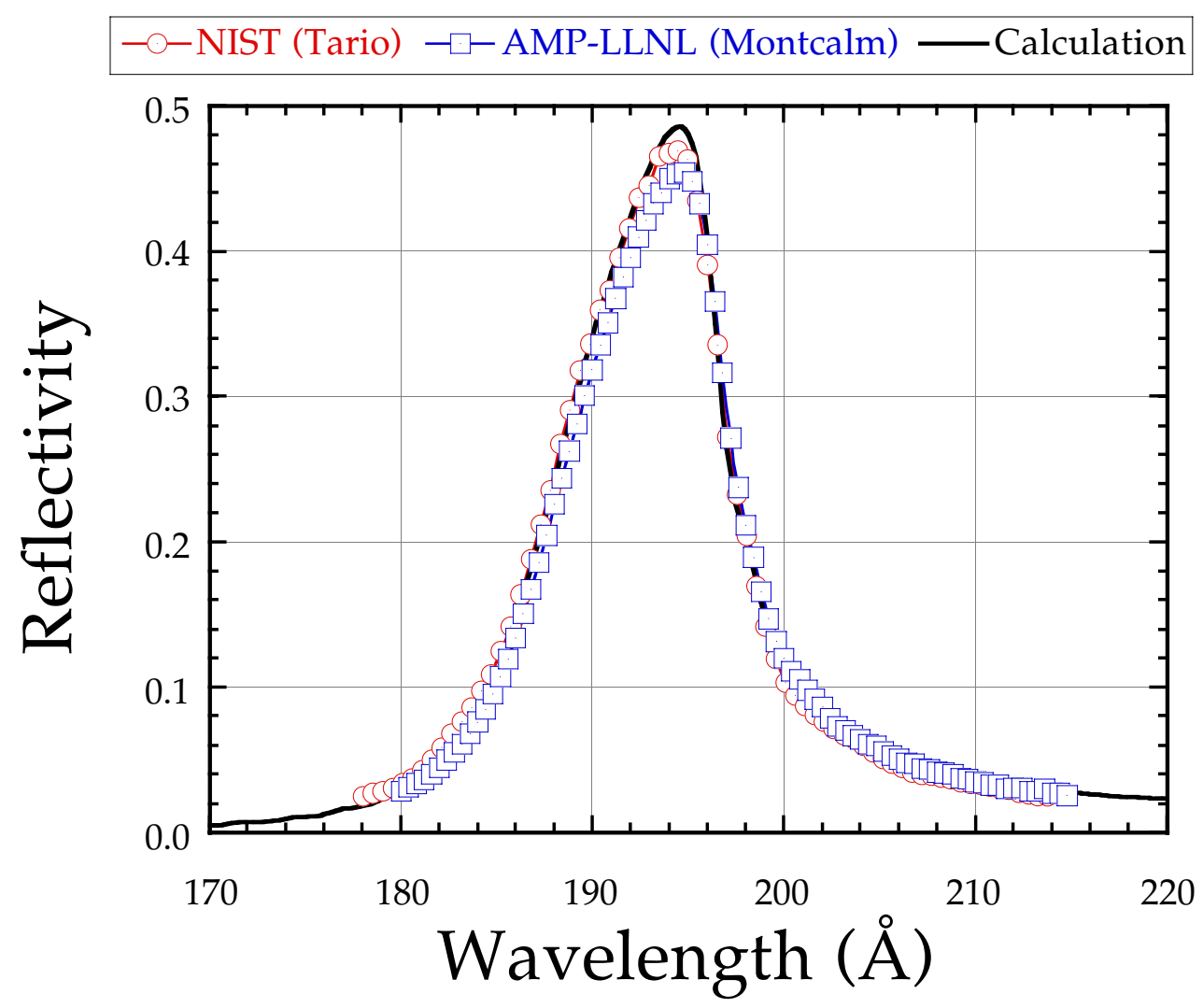

Figure 3. Experimental ( $85 \mathrm{deg}$ grazing) and calculated reflectivities of a $\mathrm{Mo}_{2} \mathrm{C}-\mathrm{Si}$ multilayer structure designed to operate at $195 \AA$ at normal incidence. The reflectivities were measured on two samples deposited on 1" dia. super polished fused silica in the same fabrication run at the National Institute of Science and Technology (Tario) and at the Advanced Light Source at Lawrence Berkeley National Laboratory (Montcalm).

photon energy at fixed angle of incidence. Note, there are white lines on the edges of Ta and W which dramatically enhance their effect on the reflectivity at those energies. The Ta $\mathrm{L}_{\mathrm{III}}$ edge energy used in the remainder of this article was determined by performing an energy scan (EXAFS) at fixed angle of incidence over the energy range $9780 \mathrm{eV}$ to $9980 \mathrm{eV}$ and detecting the total fluorescent signal as shown in Figure 4.. The white line maximum in this EXAF scan is at 9879 and is used in the remainder of this paper for the Ta edge (white line) position Measuring the reflectivity of these samples at $8979 \mathrm{eV}$ maximizes the effect of the interfacial Ta monolayers on the reflectivity and thus, on the standing wave fluorescence.

The reflectivities at $9850 \mathrm{eV}, 9879 \mathrm{eV}$ and $9950 \mathrm{eV}$ of sample 110 ( (111) $\mathrm{Si}$ substrate/C/Ta/WC/C/Ta/WC/C/Ta/...... Ta/WC/C/Ambient) are shown in Figure 5(a) and are to be 


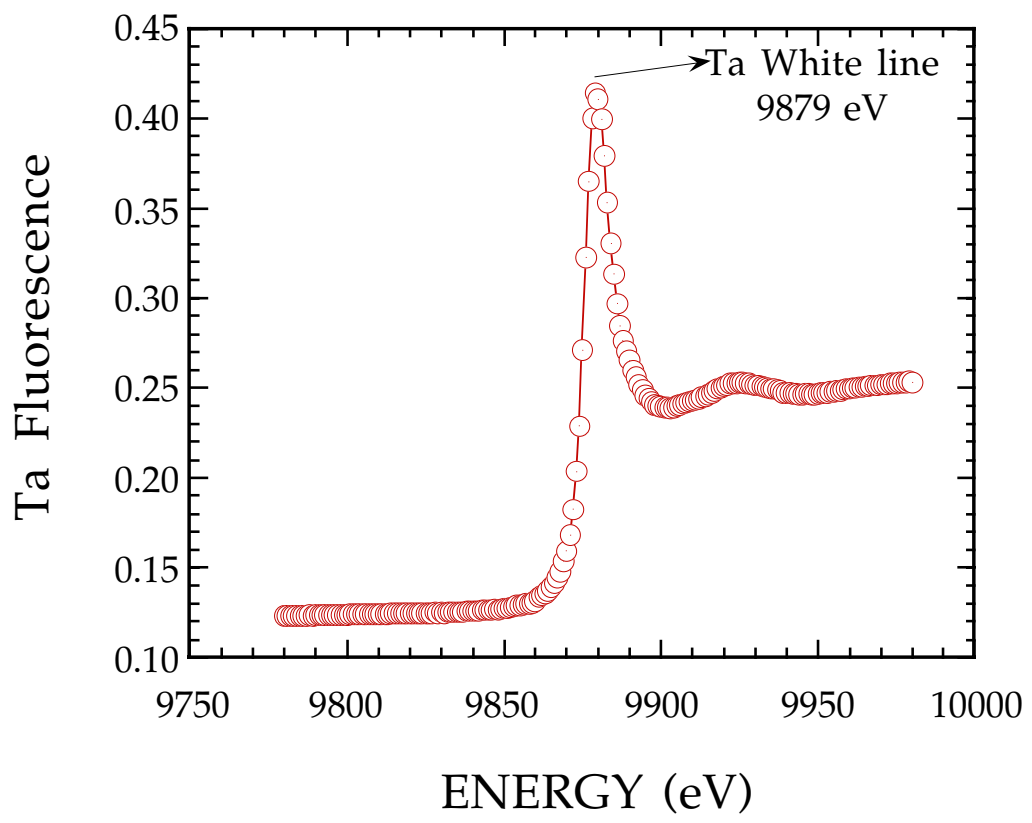

Figure 4. EXAFS scan of Sample 111 showing the Ta $\mathrm{L}_{\mathrm{III}}$ edge white line position at $9879 \mathrm{eV}$

compared with values calculated assuming chemically abrupt and atomically smooth interfaces for these same energies as shown in Figure 5(b). It is clear from these results that the effect of the interfacial Ta monolayer in this multilayer is quite small. The standing wave fluorescence data also shows that the effect of the Ta is small. Very different results are found for sample 111 where the Ta monolayer is placed at the other interface in the multilayer. The experimental reflectivities for sample 111 at $9879 \mathrm{eV}$ and $9950 \mathrm{eV}$ are dramatically decreased by the Ta monolayer as seen in Figure 6(a). Model calculations shown in Figure 6(b) give results in agreement with these observations. This indicates that the Ta monolayer layer position in sample 111 is the most effective site for damping the Bragg reflection standing wave in the multilayer. Calculations of the standing wave field support this in that in sample 111 the antinodes of the standing wave field have their maxima on the Ta monolayers.

Figure 7 shows experimental standing wave fluorescence intensities for samples 110 and 111 at $\mathrm{x}$-ray energies of $9850 \mathrm{eV}$ and $9879 \mathrm{eV}$ are shown as a function of $\mathrm{k}(=4 \pi \sin \theta / \lambda)$ in Figure 7. The SWF for these two samples are identical for $9850 \mathrm{eV}$ x-rays. At $9879 \mathrm{eV}$ sample 110 shows strong secondary extinction on the Bragg peak as expected for an efficient structure. Sample 111 exhibits a strongly attenuated primary extinction that indicates there is a strong loss mechanism operating at $8979 \mathrm{eV}$ : absorption in the Ta layer. These observations form a consistent data set demonstrating 
a) Experimental Reflectivities

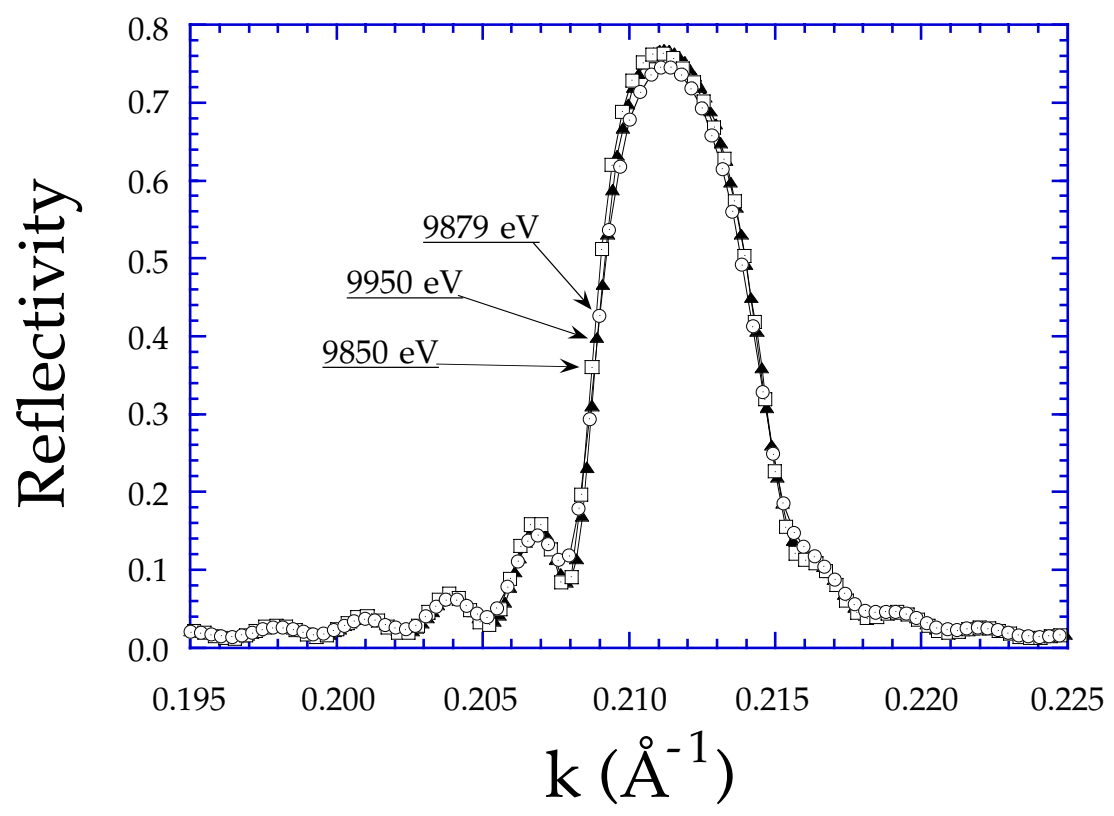

b) Calculated Reflectivities

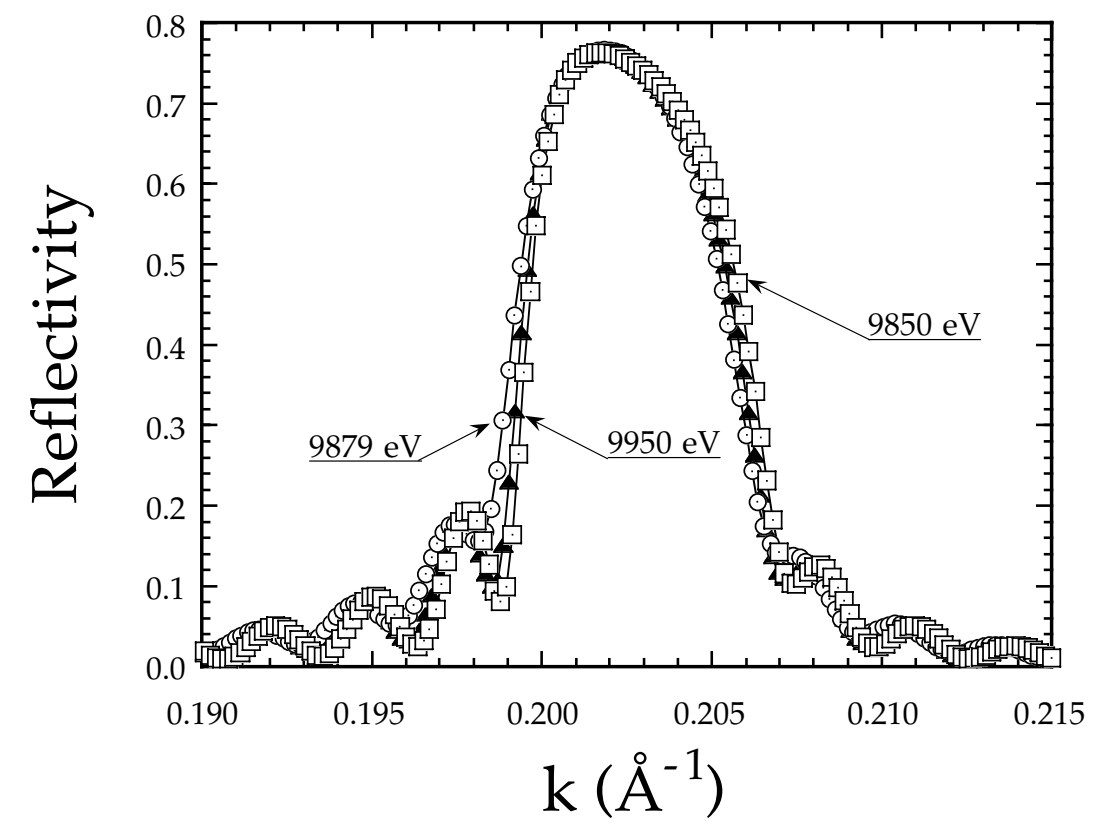

Figure 5. (a) Experimental reflectivities for sample 110 showing essentially no impact on the reflectivity due the interfacial Ta monolayer (b) Calculated reflectivities for sample 110 showing essentially no impact on reflectivity due the interfacial Ta monolayer as experimentally observed. 
a) Experimental Reflectivities

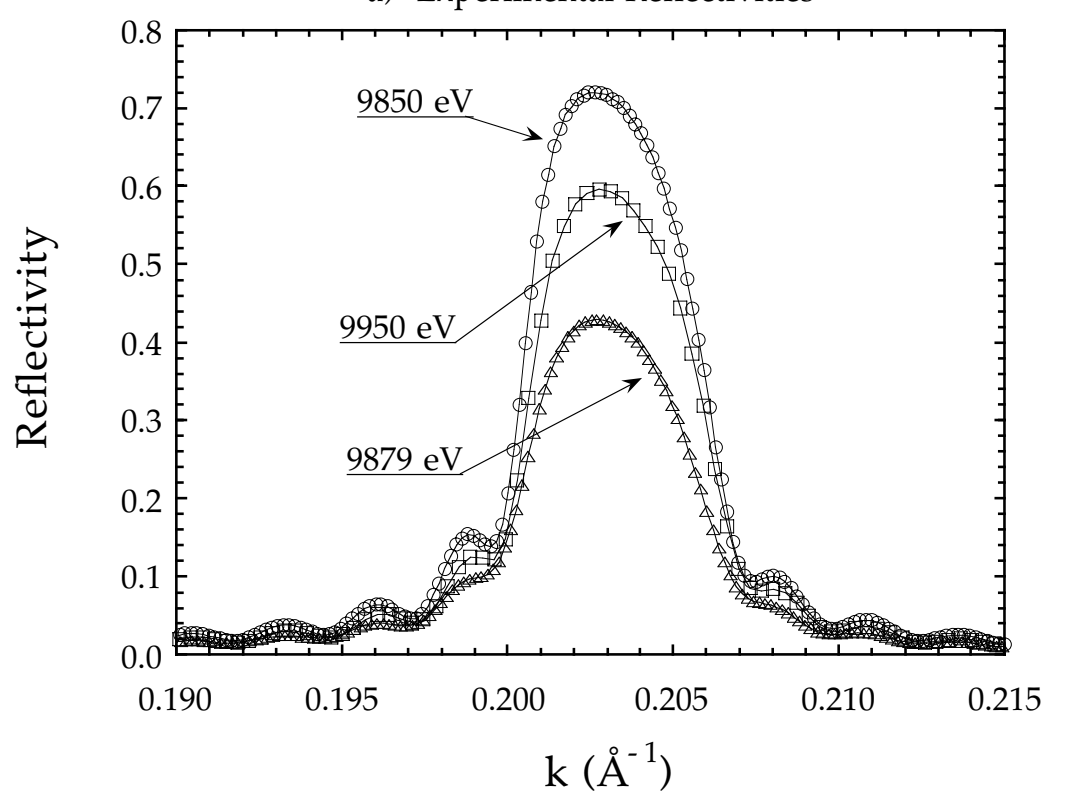

b) Calculated Reflectivities

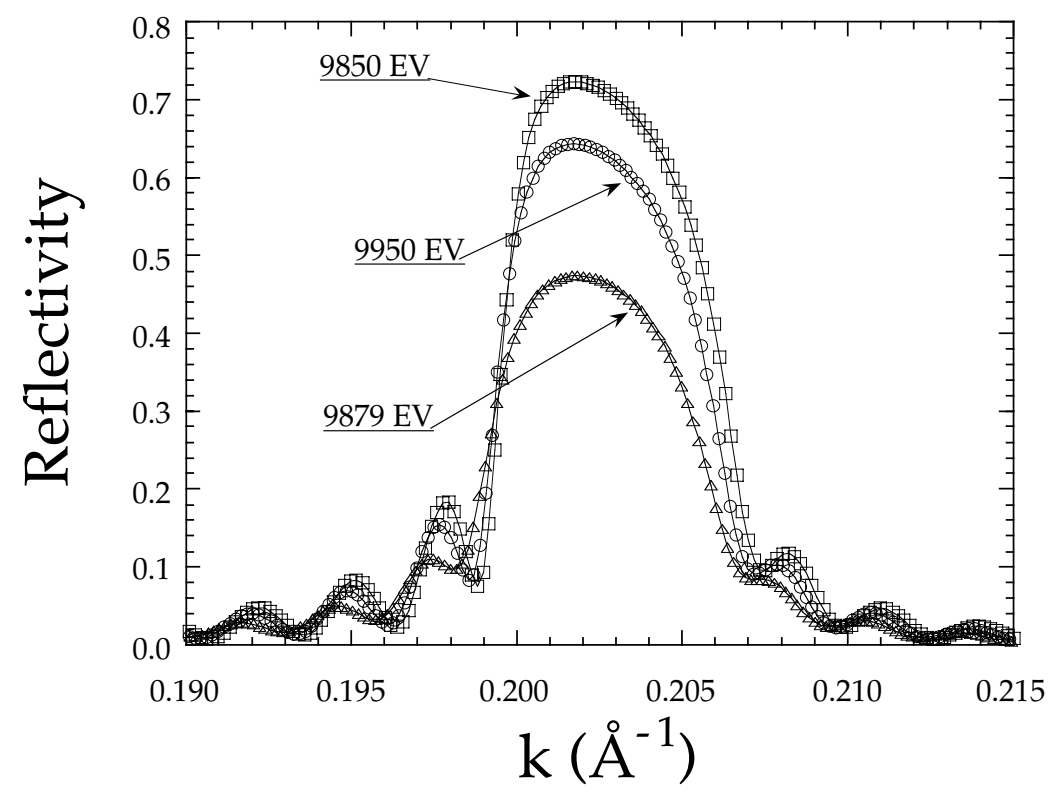

Figure 6. (a) Experimental reflectivities for sample 111 showing a dramatic impact on the reflectivity due the interfacial Ta monolayer (b) Calculated reflectivities for sample 111 showing dramatic impact on the reflectivity due to the Ta monolayer and damping the standing wave as experimentally observed. 


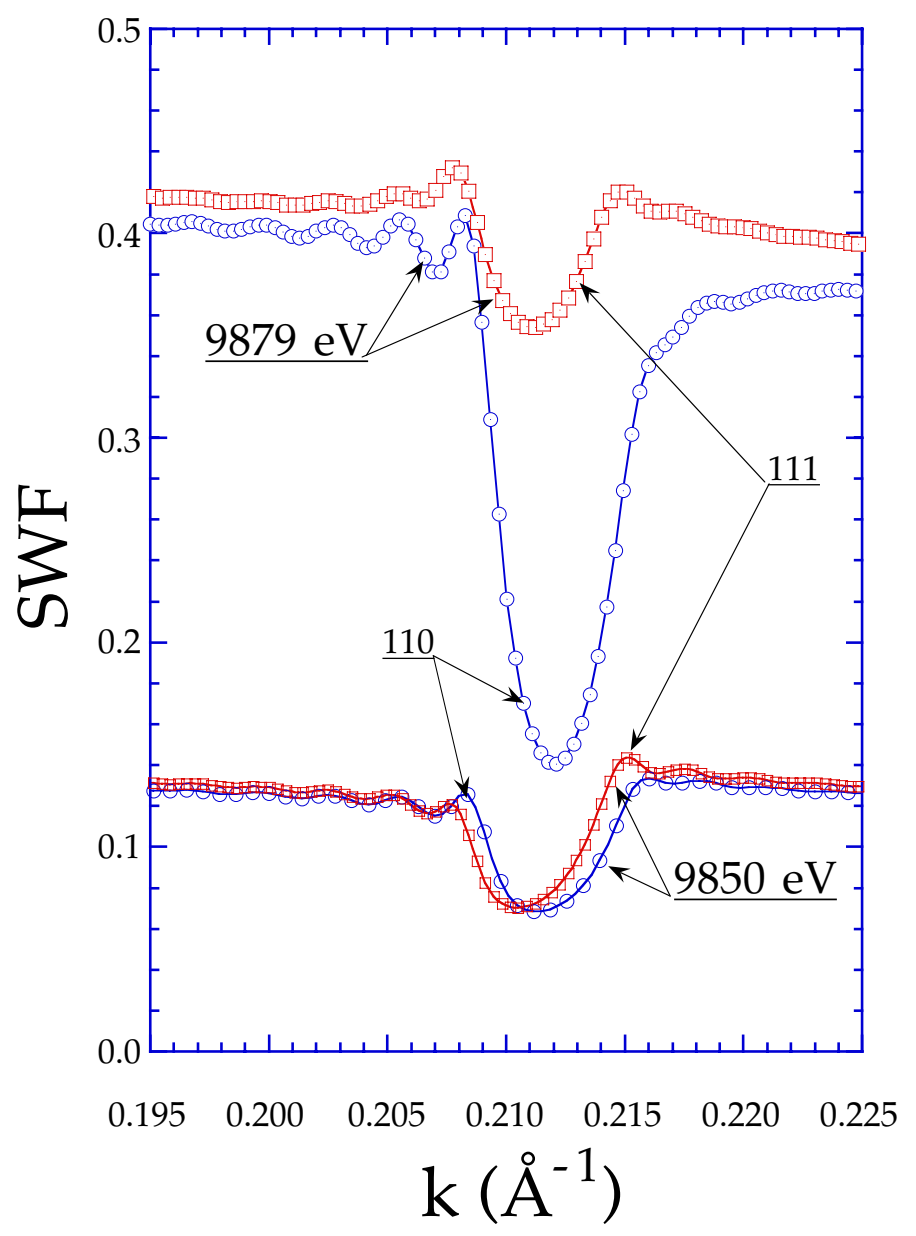

Figure 7. Experimental standing wave fluorescence intensities for samples 110 and 111 at x-ray energies of $9850 \mathrm{eV}$ and $9879 \mathrm{ev}$ are shown as a function of $\mathrm{k}(=4 \pi \sin \theta / \lambda)$. The SWF at $9850 \mathrm{eV}$ for these two samples is essentially the same for $9850 \mathrm{eV}$ x-rays. At $9879 \mathrm{eV}$ Sample 110 shows strong secondary extinction on the Bragg peak as expected for an efficient structure. Sample 111 exhibits a strongly attenuated primary extinction that indicates there is a strong loss mechanism operating at $8979 \mathrm{eV}$, absorption in the Ta layer.

that control of the structure at a specific multilayer interface site is of primary importance to performance. The general agreement between experiment and calculation indicate that model calculations can be applied to determine the relative importance of imperfections at the two interfaces characteristic of multilayer structures. 


\section{CONCLUSIONS}

One focus here is on the experimental approaches to characterizing the importance of the many structural factors that determine the performance of XR, SXR and EUV multilayer optics. Note that the optical constants of the multilayer components, substrate defects and other factors which may lead to interfacial defects are not considered. This component of the effort examines the impact of interfacial defects in multilayer structures irrespective of their origin. A second focus is on materials selection for multilayer optics again with an emphasis on interfaces. In this case the emphasis is on the properties of the materials as they are deposited one on another and the intrinsic growth characteristics of the individual layer materials themselves. Both areas are mutually complementary.

\subsection{Effect of Interfacial Chemistry}

The primary emphasis of the characterization work reported in this paper was interfaces in multilayers. The first experimental technique was limited to a very small sampling of a multilayer by the application of transmission electron microscopy to image mult-period structures in cross-section. We demonstrated that it is possible to accurately determine the incremental decrease in the multilayer period due to interface chemistry and to assess the structural and chemical quality of the interfaces formed using this approach. This approach can be generalized and applied to other material pairs as it is not material specific.

\subsection{X-ray Characterization of Interfaces in Multilayers}

The second characterization approach was developed with the intent of sampling the entire active volume in a given multilayer structure. This was selected as the goal as diffraction integrates in a complex manner over the whole volume of the sample which is illuminated. The approach taken was to essentially "TAG" the interfaces with near monolayer thick marker layers which could be selectively fluoresced by the Bragg diffraction standing wave field at appropriate $x$-ray energies. The atomic distribution of this marker layer and its planarity can be unfolded from the standing wave fluorescence data. In this work the effect of an absorbing layer at multilayer interfaces was explored as it provided the clearest information on the relative impact of imperfections at WC on C interfaces or C on WC interfaces. These experiments demonstrated that the perfection of a specific set of interfaces in a multilayer structure is primary to its performance. Additionally, the technique also provides access to information concerning interfacial roughness and atomic interdiffussion of materials. This has not been

discussed as the general quality of the WC-C multilayer was excellent so that little could be gained by a detailed analysis. 


\subsection{Summary}

It is possible to quantitatively determine the impact of chemical reactions at interfaces in mutlilayers on the multilayer period and on the structural and chemical abruptness of the interfaces by TEM characterization of specially prepared multi-period samples. Application of this approach substantially accelerated the development of the $\mathrm{Mo}_{2} \mathrm{C}-\mathrm{Si}$ multilayer system for which exceptional results are currently being achieved and is a general developmental approach for mutlilayer materials.

Chemical Tagging of interfaces enables quantitative measurement of the effects of interfacial imperfections on multilayer performance. Here we showed that imperfections at one specific set of interfaces in the WC-C system dominated the impact of interfacial imperfections on multilayer performance. This is a general result that is applicable to the design of multilayers and the development of a quantitative understanding of the effect of imperfections on multilayer performance.

\section{ACKNOWLEDGMENTS}

This work was performed under the auspices of the U.S. Department of Energy by the Lawrence Livermore National Laboratory under Contract No.W-7405-ENG-48.

\section{REFERENCES}

1. P. Dhez and C. Weisbuch, Eds., Physics, Fabrication and Applications of Multilayered Materials, (Plenum Press, New York 1988).

2. E. Spiller, Soft X-ray Optics, (SPIE Optical Engineering Press 1994).

3. T. W. Barbee, Jr., "Multilayers for X-ray Optics," Opt. Eng. 25, 898-915 (1986).

4. A. Segmuller, "Small Angle Interferences of X-rays Reflected from Periodic and Near Periodic Multilayers," in Modulated Structures - 79, J. M. Cowley et. al., Eds., AIP Conf. Proc. 53, 78-80 (1979).

5. E. Spiller, "Evaporated Multilayer Dispersion Elements for Soft X-rays," in Low Energy X-ray

Diagnostics-1981, D. T. Attwood and B. L. Henke, Eds., AIP Conf. Proc. 75, 124-130 (1981).

6. Ph. Houdy, "Kinetic Ellipsometry Applied to Soft X-ray Multilayer Growth," Revue Phys. Appl. 23, 1653-1659, (1988).

7. M. Yamamoto and T. Namioka, "In Situ Ellipsometric Study of the Optical Properties of Ultra Thin Films," Appl. Opt. 31, 1612-1621 (1992).

8. E. Spiller, "Characterization of Multilayer Coatings by X-ray Reflection," Revue Phys. Appl. 23, 1687-1700 (1988).

9. W. M. Stobbs, "Techniques for Characterizing Artificial Layer Structures Using the Fresnel Method," in Multilayers: Synthesis, Properties and Non-Electronic Applications, T. W. Barbee, Jr., F. Spaepen and L. Greer, Eds., MRS Sym. Proc. 103, 121-131 (1988). 
10. W. C. Shih and w. M. Stobbs, "Measurement of Roughness of W/Si Multilayers by the Fresnel Method", Ultramicroscopy 32, 219-239 (1990).

11. J. Slaughter, P. Kearney and C. Falco, "Characterization of Pd-B, Ag-B and Si-B Interfaces", Proc. SPIE 1547, 71-79 (1991).

12. T. W. Barbee, Jr. and W. K. Warburton, "Evanescent and Standing Wave Fluorescence from a Multilayer Structure", Materials Letters 3, 17-25 (1984). 


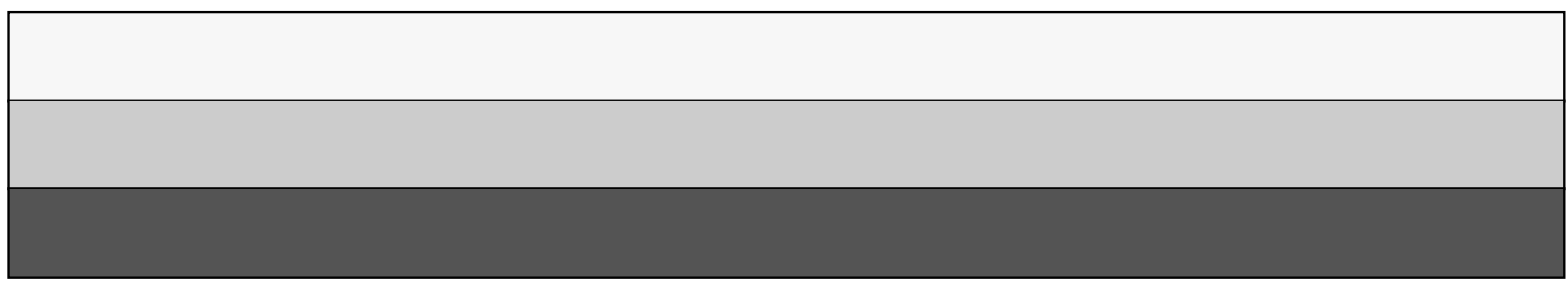

